\title{
Augmentation of EFB fiber web by nano-scale fibrous elements
}

\begin{abstract}
Treatment of the abundant oil palm empty fruit bunches with alkaline peroxide chemicals and subsequent fibrillation at varying mechanical energies resulted in favourable morphological changes of the generated fibers. The produced fibrous mass composed of intensely fibrillated elements ranging from micro to nanodiameter fibrils. nanofibrils and webs of nanofibrils were factors contributing to the functionality of the fibrous mass as fibre web augmentation elements. Profound improvement in fiber network is particularly attributable to the ability of the collected elements to fill up inter-fiber gaps and this was attributable to the micro elements in the form of micro fines, segmented micro-fibrils and webs of nanofibrils. The uniquely generated thin layers of nanofibril webs (TN-webs), were found to increase fiber web density by gluing multiple layers of fibers, together. Having landed on the surface of micro-fiber web, these TN-webs were identified as responsible for the masking effects of the underlying micro-fibres. Under such condition, fibers were observed to coalesce, suggesting also an augmented fiber network as evident from the $130 \%$ increase in tensile index and a $450 \%$ enhancement in burst index of the resultant fiber web relative to those formed with the basic alkaline peroxide chemical-mechanical refining (CMR) synergy. This reveals a great promise to EFB for application as super-strong fibre-web materials such as packaging and specialty paper-based products.
\end{abstract}

Keyword: EFB; Nano-fibrils; Fines; Thin layer nano-fiber web; TN-webs 